\title{
Cor pulmonale due to tumour embolism derived from intrasinusoidal metastatic liver carcinoma
}

\author{
R. A. BURNETT \\ From the University of Glasgow, Department of Pathology, Western Infirmary, Glasgow G11 6NT
}

SYNOPSIS Two cases of primary breast carcinoma are described, both terminating in acute cardiorespiratory failure due to multiple tumour micro-embolism. In both cases the source of the emboli was extensive metastatic intrasinusoidal carcinomatous infiltration of the liver. The association of these two uncommon conditions has not previously been reported. A brief review of the literature pertaining to diffuse intrasinusoidal metastatic carcinoma of the liver and to carcinomatous pulmonary embolism is included. The latter condition may be commoner and of greater clinical significance than has hitherto been appreciated. Some of the factors which may be responsible for this peculiar type of tumour dissemination are discussed.

Intrasinusoidal metastatic carcinoma of the liver is a relatively rare condition. In 1955, Watson reviewed the 13 cases then in the literature and added five of his own. All his cases were of bronchogenic origin. A further review by Smith (1961) expanded the number to 26 and included primary tumours from the following sites-melanocarcinoma (8), breast (7), lung (6), stomach (3), pancreas (1), and uterus (1).

In diffuse intrasinusoidal metastatic carcinoma the liver is enlarged, although the shape and architecture may be well preserved. The tumour cells diffusely infiltrate the hepatic sinusoids and are usually present in branches of the hepatic and hepatic portal veins; the liver parenchyma may be destroyed by pressure atrophy. If discrete tumour nodules. are present also, the condition is said to be of 'mixed' type (Watson, 1955). Smith (1961) noted that many of these cases showed a silent and often substantial interval between the treatment of the primary tumour and the terminal illness as well as an unusually rapid terminal course, these features being particularly prominent in cases of malignant melanoma and breast carcinoma. The diagnosis was rarely made before necropsy.

Metastatic tumour in the lungs is exceedingly common but the development of cor pulmonale on such a basis is comparatively rare. In 1961, Durham et al reviewed the 17 cases then reported and added one of their own. Three main groups

Received for publication 13 December 1974 were delineated as previously described by Storstein (1951).

Type a: lymphangitis carcinomatosa, the pulmonary lymphatic vessels being extensively invaded by tumour with compression of alveoli and capillaries and loss of lung compliance Type b: lymphangitis carcinomatosa with compression and invasion of small arteries and arterioles producing thrombosis and/or endarteritis

Type c: a purely embolic form in which the pulmonary arteries and arterioles are occluded by cancerous emboli, usually with secondary thrombosis and/or endarteritis.

Considerable overlap may be present between these three types but essentially two main groups, the lymphatic and the embolic, may be distinguished. Stomach (Morgan, 1949) and breast (Mason, 1940) were the commonest primary sites, accounting between them for the majority of the reports in the literature at that time. More recently, other tumours have been implicated, particularly chorionepithelioma (Bagshawe and Brooks, 1959) and hepatoma (Winterbauer et al, 1968).

The embolic group (type c) in its pure form is the most clear cut entity, not requiring the presence of previous lung tumour or lymphatic spread and resulting in a dramatic clinical picture because of its purely vascular involvement. Of the 18 cases of Durham et al (1961), eight were purely or predominantly of this type. Winterbauer et al (1968) assembled 35 cases all of the embolic form (type c), 
in each of which the tumour embolism was considered to be the immediate cause of death. These included stomach, breast, chorionepithelioma, hepatoma, and one case each with thyroid and parotid primaries. Unfortunately, these authors also included entities of doubtful malignancy in their series, including a case of trophoblastic embolism and several of hydatidiform mole.

Although fatal cor pulmonale due to tumour embolism is rare, minor degrees of embolism are not uncommon. Willis $(1952$, p. 39$)$ points out that 'Frequently in cases with disseminated tumours, microscopical study of apparently unaffected areas of lung discloses pulmonary arterioles occluded by tumour thrombi'. The findings of Winterbauer et al (1968) support this observation and also suggest that the incidence of clinically significant degrees of embolism is much greater than has been previously appreciated. The consequences of tumour impaction in the pulmonary circulation, namely thrombosis and reactive endarteritis, are described by Saphir (1947), and the entire subject is reviewed in Revista Clinica Espanola (Editorial, 1969).

\section{Case reports}

CASE 1

The patient presented in September 1972 at the age of 41 years with a six-week history of a painful lump in the left breast. Her past medical history included negative investigations for thyrotoxicosis in 1969 and a history of infertility; she also suffered from severe congenital kyphoscoliosis. She smoked 60 cigarettes per day.

The mass was excised three days later and frozen section confirmed the clinical diagnosis of carcinoma. Simple mastectomy was performed under the same anaesthetic, the axilla being left undisturbed and a course of postoperative radiotherapy given. Microscopy of the tumour mass showed extensive intraduct carcinoma of comedo type and infiltrating, moderately differentiated, metaplastic squamous cell carcinoma (fig 1). Keratinization was evident in the tumour, and in a few areas intercellular bridges could be demonstrated. Extensive lymphatic invasion was evident.

The patient remained well for 15 months, until January 1974 when she was readmitted in extremis. Five days before admission she had experienced dyspnoea of sudden onset. This was at first rather intermittent but became progressively worse until she was severely and continuously breathless at rest. On examination she was pale, slightly jaundiced, and extremely dyspnoeic. The pulse was 120 per minute and regular, and the jugular venous pressure was markedly raised. There was no clinical evidence

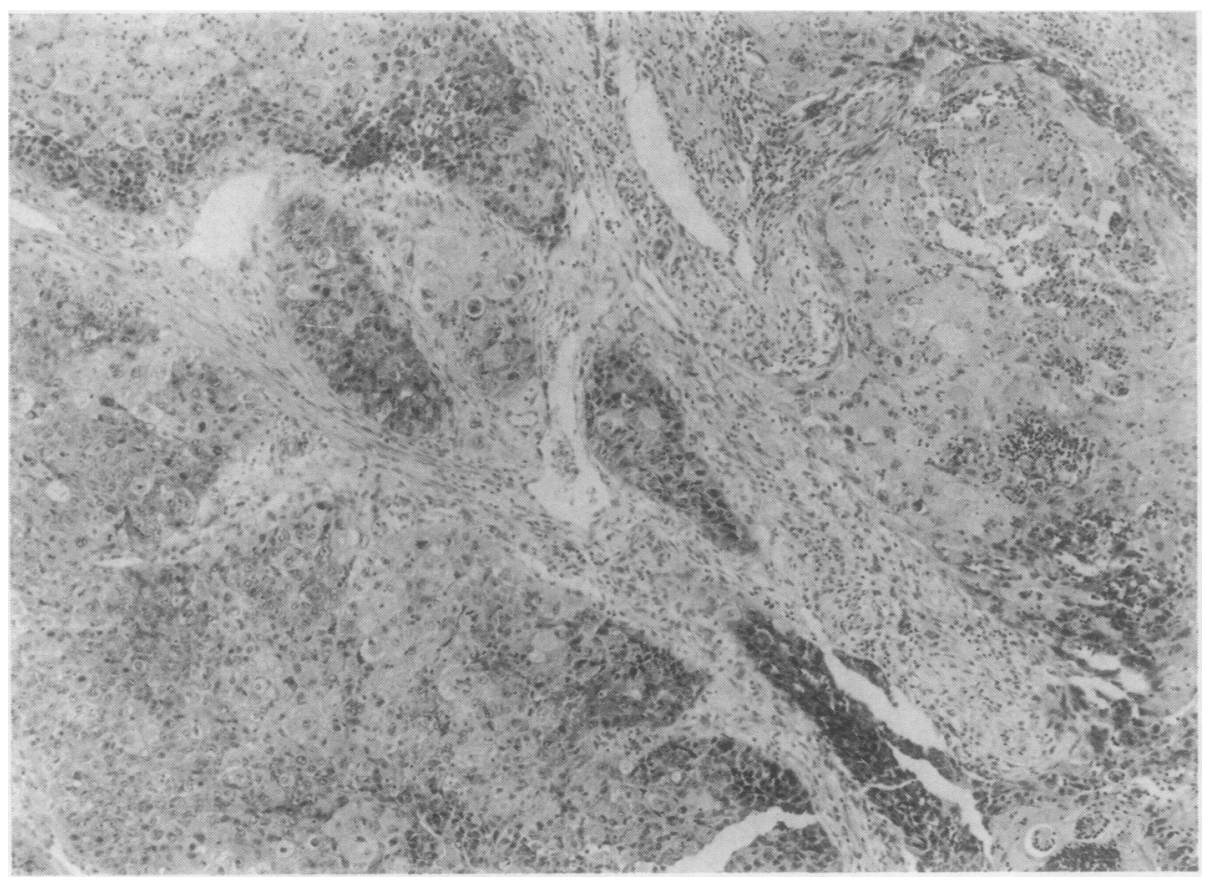

Fig 1 Case 1. The primary breast tumour. Haematoxylin and eosin $\times 60$. 
of metastatic disease. Chest radiography showed no change from the previous films of 1972. She died shortly afterwards.

\section{Necropsy}

The body was that of a slightly icteric adult female. An old left mastectomy scar was present, and kyphoscoliosis affecting the lumbar and dorsal spine was noted. The heart $(230 \mathrm{~g})$ showed rightsided dilatation but no hypertrophy. The left pleural space was obliterated by dense old adhesions, possibly relating to her radiotherapy. The lungs (right, $600 \mathrm{~g}$; left, $480 \mathrm{~g}$ ) were congested and showed patchy areas of collapse. The large pulmonary arteries were patent throughout. The liver (1430 g) appeared pale and firm. A large proportion of this organ was streaked with haemorrhagic areas and appeared necrotic. Numerous small whitish nodules of secondary tumour were present, particularly in the right lobe. Tumour metastases were present in the lumbar spine and also within mediastinal lymph nodes. There were no other significant findings.

\section{Microscopy}

As well as the tumour nodules noted at necropsy, the liver showed extensive intrasinusoidal infiltration by anaplastic carcinoma, almost the whole liver substance being involved (figs 2 and 3). The appearances were consistent with a breast carcinoma but, unlike the primary tumour showed little tendency towards squamous differentiation. In many areas there was only mild parenchymal damage with intrasinusoidal tumour columns infiltrating through an intact liver architecture. Elsewhere compression and atrophy of the liver cords had occurred to the point where they could be recognized only as linear deposits of lipofuscin pigment lying between parallel tumour columns, the reticulin framework being preserved. Little desmoplastic reaction was present in these areas of intrasinusoidal tumour but this became more prominent with the coalescence of the tumour to form the macroscopically identifiable nodules. There was widespread involvement of branches of both the hepatic and hepatic portal veins by tumour plugs, sometimes with associated thrombus. Invasion of branches of the hepatic artery was not seen. Haemorrhagic areas were also present, particularly in the coalescing intrasinusoidal tumour areas. Inflammatory cell response was not prominent, being restricted to areas of tumour necrosis and to occasional foci of round cells, particularly around vessels.

The lungs showed a striking picture of vascular occlusion by anaplastic tumour. The majority of the small pulmonary arteries in both lungs contained viable tumour emboli, and tumour was also extensively present in arterioles, venules, and, less frequently, in larger veins. Occasionally single tumour cells or short columns were seen in alveolar capillaries. Very little thrombosis or reactive endarteritis was present in the pulmonary vessels but accumulations of fibrin were frequently seen, particularly in the arterioles, either related to tumour cells or occluding the vessel in the absence of demonstrable tumour. There was no parenchymal invasion but occasional lymphatic vessels containing tumour plugs were noted. The intravascular tumour plugs showed occasional mitotic figures, and in the larger ones some central necrosis was present. The lungs were markedly congested with dilatation of the larger arteries and veins and areas of lung collapse. In addition to the acute changes noted above there was evidence of chronic pulmonary hypertension, and much dust was also present. These changes may be related respectively to the patient's kyphoscoliosis and to her smoking. The metastases in the mediastinal lymph nodes and lumbar spine were confirmed and they consisted of similar anaplastic carcinoma.

\section{CASE 2}

The patient first presented in April 1972, aged 35 years, with discrete lumps in the left breast. Excision biopsy was performed and rather unexpectedly histology revealed a primary anaplastic breast carcinoma (fig 4) with evidence of lymphatic invasion. She was seen again two months later when induration below the scar and a hard, mobile lump in the left axilla were noted. Simple mastectomy with axillary clearance was performed in August 1972, and a course of postoperative radiotherapy was given.

The mastectomy specimen contained a $2 \mathrm{~cm}$ diameter tumour nodule close to the nipple and adjacent to a haematoma which had formed deep to the original biopsy scar. Several lymph nodes were included with axillary fat. Microscopy showed extensive cribriform intraduct carcinoma and scirrhous infiltrating carcinoma, a few areas of which showed attempts at adenocarcinomatous differentiation. The lymph nodes showed almost complete replacement by similar tumour.

The patient remained well until February 1974, when she returned with two small firm swellings in the left side of the neck. Excision biopsy was performed and frozen section confirmed that they were lymph nodes which had been replaced by anaplastic tumour. On the basis of the frozen section reports, bilateral oophorectomy was performed, and in the abdomen the surgeon noted the 


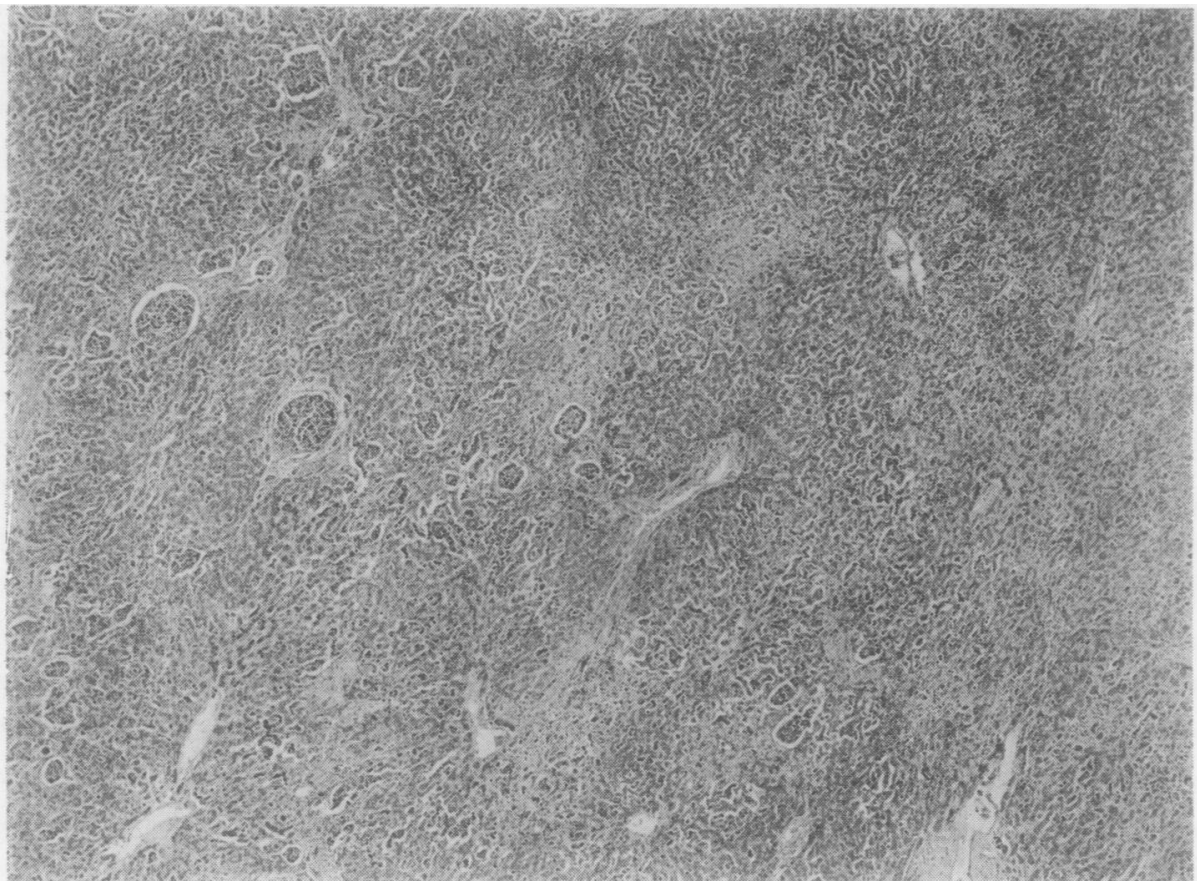

Fig 2 Case 1. Low-power view of the liver showing intrasinusoidal tumour. Note the extensive venous invasion. $H$ and $E \times 24$.

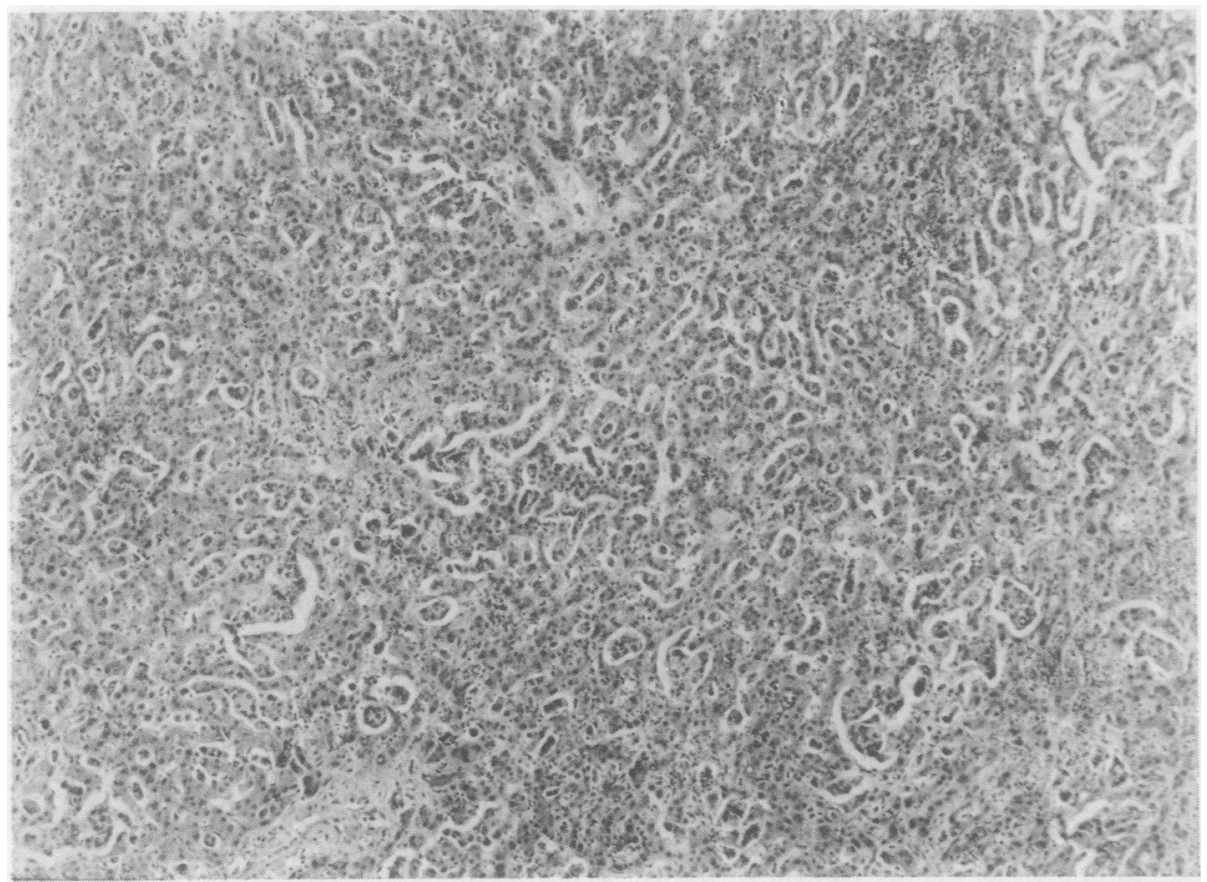

Fig 3 Case 1. Higher-power view of a similar area to that shown in fig 2 shows diffuse intrasinusoidal tumour. $H$ and $E \times 60$. 


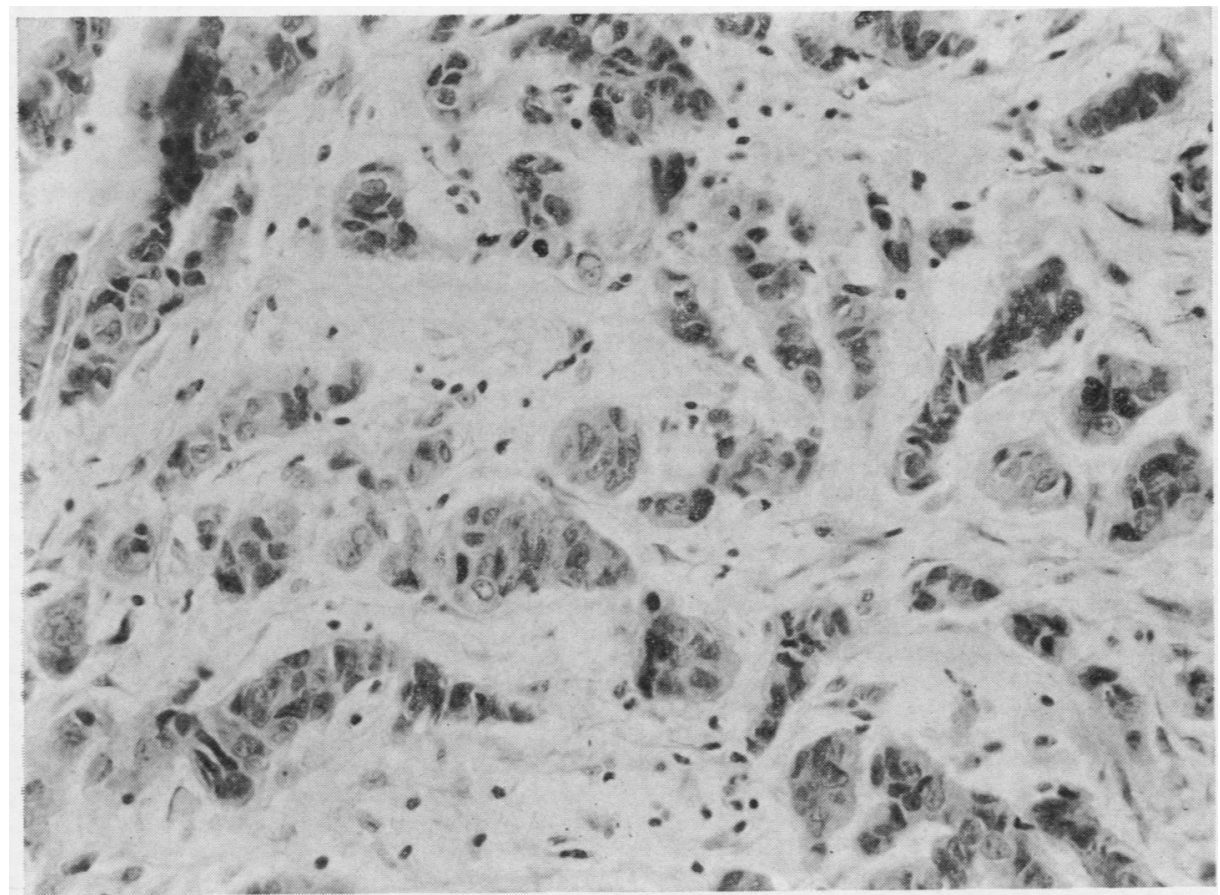

Fig 4 Case 2. The primary breast tumour. $H$ and $E \times 240$.

absence of any tumour in either the peritoneal cavity or liver. When next seen in April 1974, the patient was complaining of back pain and radiography showed secondary tumour in the lumbar spine and pelvis. She was treated with palliative spinal radiotherapy followed by a course of cytotoxic chemotherapy. At that time the liver was thought to be enlarged. She was finally admitted in June 1974 with a four-day history of increasing dyspnoea and also anorexia, nausea, and vomiting, which she attributed to the chemotherapy. On examination she was distressed and markedly breathless at rest.

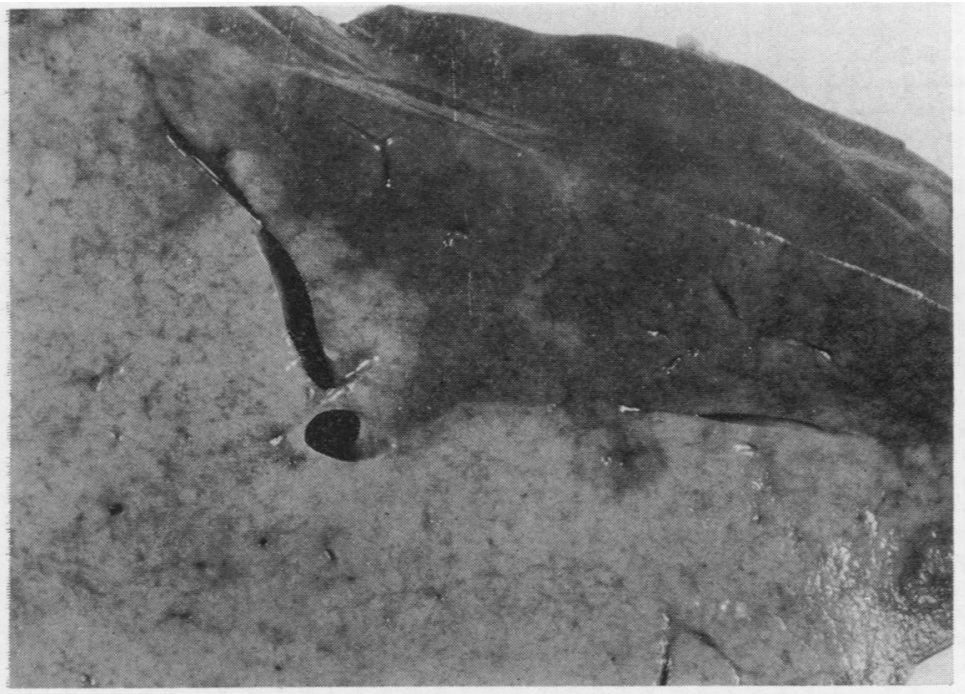

Fig 5 Case 2. Close-up of the gross appearance of the liver. The lighter area corresponds to intrasinusoidal spread with parenchymal atrophy; the darker area is less heavily involved and atrophy is minimal. Note the preservation of architectural markings in both areas. 


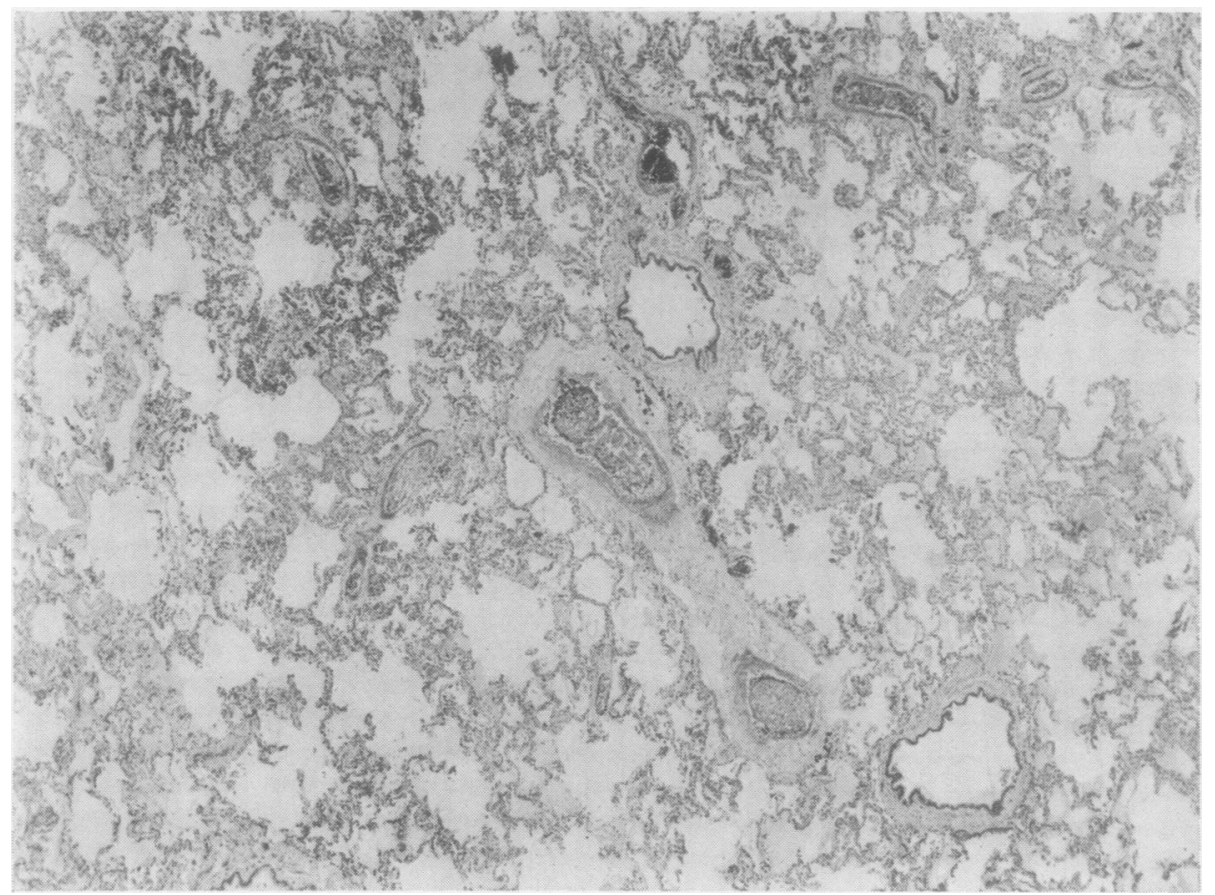

Fig 6 Case 2. Almost all the arterial vessels in this lung field are occluded by tumour plugs. There is neither thrombosis nor endarteritis. $H$ and $E \times 23$

A degree of jaundice was noted. Examination of the lung fields revealed no abnormality but a degree of smooth hepatomegaly was present. A chest radiograph was entirely normal. She rapidly became hypotensive and died shortly after admission.

\section{Necropsy}

The body was that of an adult female. Old left mastectomy and transverse suprapubic scars were present. The heart $(300 \mathrm{~g})$ showed right-sided dilatation but no hypertrophy. The lungs (left, $500 \mathrm{~g}$; right, $500 \mathrm{~g}$ ) were congested but showed no other abnormality. The pulmonary arteries appeared patent throughout. The liver $(2600 \mathrm{~g})(\mathrm{fig} \mathrm{5})$ was enlarged, pale, and smooth. The right lobe was completely replaced by whitish tumour with small areas of infarction. The left lobe was markedly congested with accentuation of the lobular pattern and contained occasional tumour nodules. The intrahepatic branches of the portal vein appeared to be thrombosed, and the major hepatic veins were also occluded. Small nodules of tumour were present in the right adrenal gland and in the cortex of the left kidney. Extensive metastatic tumour was present in the lumbar spine.

\section{Histology}

The right lobe of the liver showed almost complete carcinomatous replacement by intrasinusoidal anaplastic tumour, the liver parenchyma being destroyed but the lobular and reticulin architecture remaining. The left lobe showed patchy intrasinusoidal spread without significant parenchymal atrophy or destruction but with marked vascular invasion. No desmoplastic reaction was present in $\frac{7}{0}$ either lobe. Numerous branches of the hepatic portal and hepatic veins contained tumour plugs, $N$ sometimes associated with thrombus. Tumour was $N$ not seen within branches of the hepatic artery. Mitoses were quite frequent but tumour necrosis was not present and the inflammatory cell response was minimal. Cholestasis was marked. The lungs showed a histological picture of carcinomatous $₫$ embolism virtually identical with that of case 1 (fig 6). These lungs however showed no evidence of pre-existing disease, and no tumour was present in the pulmonary lymphatic vessels. A slightly $\frac{?}{0}$ greater degree of fibroblastic endarteritis was $\bigcirc$ present in the pulmonary arterioles than in case 1,2 although this was still not marked and quite difficult 8 to find. No thrombosis was present. Metastatic 
tumour was confirmed in the lumbar spine, right adrenal, and left kidney, and in these sites the adenocarcinomatous component of the tumour was more prominent with areas of mucin production. No desmoplastic reaction was noted in these metastases.

\section{Discussion}

Metastatic intrasinusoidal liver carcinoma and cor pulmonale due to tumour micro-embolism are rare conditions. Primary carcinomata of the stomach and breast are capable of giving rise to both. The occurrence together of these two conditions has not previously been reported but the association does not seem particularly surprising-especially when associated with a primary from one of the above sites-because invasion of the hepatic veins is almost invariable in intrasinusoidal carcinoma of the liver (Watson, 1955). Willis (1952, p. 186) states: 'In nearly all cases in which I have observed hepatic venous invasion, numerous pulmonary metastases also were present. In one such instance, in which the lungs apppeared normal, microscopical search revealed cancerous thrombi in pulmonary arterioles'. The embolic nature of this phenomenon is well illustrated in the report of Storey and Goldstein (1962), in which invasion of the main hepatic veins by a malignant hepatoma was followed by massive tumour embolism to the lungs. Winterbauer et al (1968) present a good case for relating the incidence of tumorous embolism to the degree of liver involvement by metastatic carcinoma and particularly to the degree of hepatic venous invasion.

Excluding the melanocarcinomas, all of the 17 cases of intrasinusoidal liver carcinoma reported by Smith (1961) were undifferentiated tumours, and this correlates well with the work of Densert and Söderström (1965), who reviewed diffuse metastases from primary bronchogenic carcinoma to the liver and spleen in a necropsy series. These workers found an overall incidence of $2.6 \%$ of diffuse liver involvement but this occurred only with 'smallcelled' types of bronchial carcinoma.

Oertel (1935) described a case (type c) of gastric carcinoma in which infiltration of the hepatic sinusoids was associated with widespread plugging of pulmonary and peribronchial arteries, veins, and lymphatics by tumour emboli, but he makes no mention of cor pulmonale.

The first specific reports of cor pulmonale caused by tumour were those of Brill and Robertson (1937) (type b) and Mason (1940) (type c), the primary sites being in the stomach and breast respectively. In both these papers the term 'subacute cor pulmonale' was used to delineate this apparently specific clinicopathological entity, and Morgan (1949) also used the term in this way in his review of lymphangitis carcinomatosa. This view was challenged by Durham et al (1961), who argued that the pathogenesis of cor pulmonale due to carcinomatous embolism was in no way different from the more common forms of pulmonary embolism. Altemus and Lee (1967) commented that subsequent reports have emphasized that non-malignant emboli, originating from systemic veins or uterine trophoblastic elements, may initiate an identical clinical picture. Certainly neither of the cases reported here, with terminal illnesses of five and four days respectively, could be included under this heading because Mason (1940) defined subacute cor pulmonale as having a course of between nine days and two months.

The case reported by Durham et al (1961) was similar in most respects to the two reported here (type c). Their patient, a 39-year-old woman who had undergone radical mastectomy and radiotherapy for carcinoma nine months previously, presented with a three-week history of progressive dyspnoea. There was extensive tumour embolism to the smaller pulmonary arterial vessels, some of which also contained thrombus. Others showed a fibroblastic intimal proliferation around clumps of degenerating tumour cells. The liver weighed $2000 \mathrm{~g}$, and most of the parenchyma was replaced by irregular greyish to yellowish white tumour tissue with areas of necrosis. Microscopy showed extensive invasion of hepatic veins by tumour. There was no mention of an intrasinusoidal mode of spread within the liver, although this seems possible from the description.

The two cases described in this paper show remarkable similarities, particularly in the acuteness of the terminal illnesses. The histology of the lungs, particularly the absence of thrombosis and the scanty and poorly developed reactive endarteritis, correlates well with the duration of dyspnoea. In most of the cases previously described these latter changes were more prominent because the patients survived for much longer. The absence of right ventricular hypertrophy also supports the concept of a shower of emboli occurring over a few days, rather than a gradual process which would have resulted in chronic pulmonary hypertension. A degree of pulmonary hypertension was present in the first case, being almost certainly related to the kyphoscoliosis (Bergofsky et al, 1959) and not to the tumour embolism, but there was no evidence of previous episodes of cardiac decompensation. The paucity of clinical signs in the chest and the 
absence of radiological changes in both cases are in accord with the findings of previous workers in type (c) cases (Altemus and Lee, 1967; Winterbauer et al, 1968).

Neither of these cases showed the epigastric pain and abdominal swelling which Watson (1955) described as being frequently seen in intrasinusoidal liver carcinoma, but both were jaundiced, this being more common than with the nodular forms of metastatic liver tumour. Clinically, in both cases, the cause of the terminal cardiorespiratory failure was completely obscure, and in the first case necropsy did not elucidate the problem as the gross appearances were misinterpreted. Oertel (1935) also failed to diagnose intrasinusoidal hepatic tumour on the gross appearances in his case, and Watson (1955) comments on the problem. The case of intrasinusoidal carcinoma reported by Cracium et al (1931), where the primary was a scirrhous breast tumour, is of interest. Apparently the diffusely infiltrating tumour cells in the liver had retained their capability to stimulate a desmoplastic reaction, and the gross appearance of the liver was indistinguishable from that of diffuse hepatic fibrosis. Micolonghi et al (1958) reported a similar case, and again the necropsy diagnosis was 'cirrhosis', the presence of tumour being found only on microscopical examination. That the cirrhotic appearance of the liver was due to the tumour and had not preceded it was excluded by these authors on morphological grounds. Lieber (1957) pointed out that tumour metastasis to a cirrhotic liver is unusual, and in his series of 24329 cancer deaths it occurred in only $0.12 \%$ of cirrhotic livers, compared to $28.6 \%$ of non-cirrhotic organs. A cirrhotic appearance was seen in neither of the present cases. In case 2 the presence of diffuse hepatic tumour was appreciated at necropsy and the invasion of the hepatic veins was correctly interpreted. It was then possible to predict with some confidence the histological appearance of the lungs, in view of the clinical history and in the absence of any naked-eye changes. Four months previously, during the oophorectomy operation, this liver had been visualized by the surgeon and was considered to be free of tumour. It is worth mentioning that in the absence of specific contraindications the diffuse nature of intrasinusoidal metastatic carcinoma makes this condition highly susceptible to diagnosis by needle biopsy of the liver.

\section{References}

Altemus, L. R. and Lee, R. E. (1967). Carcinomatosis of the lung with pulmonary hypertension. Arch. intern. Med.0 119, 32-38.

Bagshawe, K. D. and Brooks, W. D. W. (1959). Subacute $\overrightarrow{0}$ pulmonary hypertension due to chorionepithelioma:Lancet, 1, 653-658.

Bergofsky, E. H., Turino, G. M., and Fishman, A. P. (1959). Cardiorespiratory failure in kyphoscoliosis. Medicineo (Baltimore), 38, 263-317.

Brill, I. C. and Robertson, T. D. (1937). Subacute cori pulmonale. Arch. intern. Med., 60, 1043-1057.

Cracium, E. C., Aslan, A., and Caffé, L. (1931). Cirrhosè atrophique néoplasique secondaire. (Quoted by Smith, ㄱ 1961). Ann Anat. path., 8, 1089-1112.

Densert, O. and Söderström, J. (1965). Diffuse metastases in bronchial cancer. Acta path. microbiol. scand., 64, 477-484.

Durham, J. R., Ashley, P. F., and Dorencamp, D. (1961). Cor pulmonale due to tumor emboli. J. Amer. med. Ass., 175, 757-760.

Editorial 1969. Tumoral pulmonary embolism. Rev. clin. जे Esp., 113, 540-541.

Lieber, M. M. (1957). The rare occurrence of metastatic carcinoma in the cirrhotic liver. Amer. J. med. Sci., 233, 145-152.

Mason, D. G. (1940). Subacute cor pulmonale. Arch. intern. Med., 66, 1221-1229.

Micolonghi, T., Pineda, E., and Stanley, M. M. (1958). 요 Metastatic carcinomatous cirrhosis of the liver: report of a $\overrightarrow{\vec{A}}$ case in which death followed hemorrhage from esophageal윽 varices and hepatic coma. Arch. Path., 65, 56-62.

Morgan, A. D. (1949). The pathology of subacute cor pulmonale in diffuse carcinomatosis of the lungs. J. Path. Bact., 61, 75-84.

Oertel, H. (1935). On a peculiar vascular transportation ando generalization of carcinoma without local metastases. 0 J. Path. Bact., 40, 323-334.

Saphir, O. (1947). The fate of carcinoma emboli in the lungs. Amer. J. Path., 23, 245-253.

Smith, J. C. (1961). Diffuse intrasinusoidal metastatic cancer of the liver. Ann. intern. Med., 54, 104-113.

Storey, P. B. and Goldstein, W. (1962). Pulmonary embolization from primary hepatic carcinoma. Arch. intern. Med., 110, 262-269.

Storstein, O. (1951). Circulatory failure in metastatic N carcinoma of the lung: a physiologic and pathologic study of its pathogenesis. Circulation, 4, 913-919.

Watson, A. J. (1955). Diffuse intra-sinusoidal metastatic N carcinoma of the liver. J. Path. Bact., 69, 207-217.

Willis, R. A. (1952). The Spread of Tumours in the Human Body. 2nd edition. Butterworth, London.

Winterbauer, R. H., Elfenbein, I. P., and Ball, W. C. Jr. (1968). Incidence and clinical significance of tumor embolisation to the lungs. Amer. J. Med., 45, 271-290. 